\title{
Recombinant Insulin-Like Growth Factor
}

National Cancer Institute

\section{Source}

National Cancer Institute. Recombinant Insulin-Like Growth Factor. NCI Thesaurus. Code C835.

A recombinant human antiapoptotic growth factor. Recombinant insulin-like growth factor (IGF) binds to and activates the insulin and IGF receptors; activation of these receptors initiates intracellular phosphorylation events and the activation of several signaling pathways, thereby promoting cellular proliferation and inhibiting apoptosis. High levels of circulating endogenous insulin-like growth factor may indicate a poor prognosis in some cancers. ( $\mathrm{NClO4})$ 